\title{
Control of Grain Boundaries in Orthorhombic Alpha-Uranium
}

\author{
D. A. Carpenter*, J. L. Pugh*, and D. F. Teter** \\ * Y-12 National Security Complex, Box 2009, Oak Ridge, Tennessee, 37831-8084 \\ ** Los Alamos National Laboratory, Box 1663, Los Alamos, New Mexico, 87545
}

Depleted $\alpha$-uranium is an orthorhombic metal that has a variety of commercial and military uses. In some cases, mechanical property requirements might benefit from attempts to control the grainboundary distribution through thermomechanical processing. Gertsman suggested that special boundaries in non-cubic materials are probably twin boundaries, since in these boundaries, all atoms from the two grains are exactly coincident.[1] Thus, where calculations such as that of Bonneau and Cousineau[2] reveal near-coincident-site lattices (NCSL) and associated sigma values, atoms in twin boundaries extracted from these NCSL's may be shifted into exact coincidence. To a first approximation, the planar densities of these boundaries might be taken to represent the relative energies of the boundaries. Figure 1 illustrates a relatively dense, hypothetical twin boundary based upon a $\Sigma 4$ NCSL, formed by a $60^{\circ}$ rotation about the [010] vector. The boundary plane is the (101) plane.

Orientation Imaging Microscopy (OIM) data were collected from several specimens of $\alpha$-uranium that had been subjected to a TMP schedule designed to produce fine-grain metal. Variations in the parameters of the TMP schedule resulted in material having average grain sizes ranging from 10 to $40 \mu \mathrm{m}$ and variable mechanical properties. The OIM data indicated preferred orientations, as well as a tendency for some material to favor special boundaries. Figure 2 illustrates a typical inverse pole figure (IPF) map of the wrought material. The pole figure in Fig. 3 and the texture-reduced, misorientation distribution function (MDF) in Fig. 4 were determined from a combination of several wide area scans that averaged approximately 1-2 points per grain. The enhancement of intensity about the $60^{\circ} /$ [010] misorientation suggested enhancement of stable boundaries associated with the $\Sigma 4$ NCSL related to that misorientation. More work is in progress to determine how to optimize the enhancement of special boundaries.

\section{References}

1. V. Y. Gertsman and J. A. Szpunar; Materials Science Forum, vols. 294-296, pp. 181-186 (1999). Trans Tech Publications, Switzerland, 1999.

2. The Y-12 National Security Complex is managed by BWXT-Y12, L.L.C., for the U. S. Department of Energy, under contract number DE-AC05-00OR22800. 


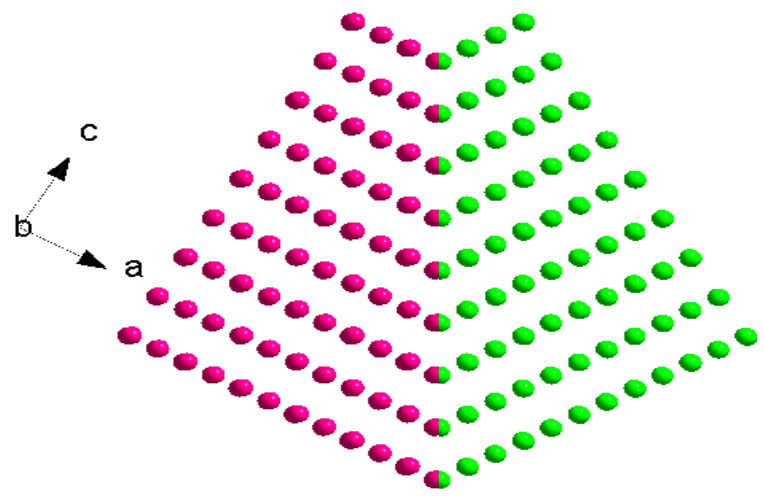

FIG 1 Hypothetical grain boundary based upon the (101) twin plane from the $\Sigma 4$ NCSL with misorientation $60^{\circ} /[010]$.
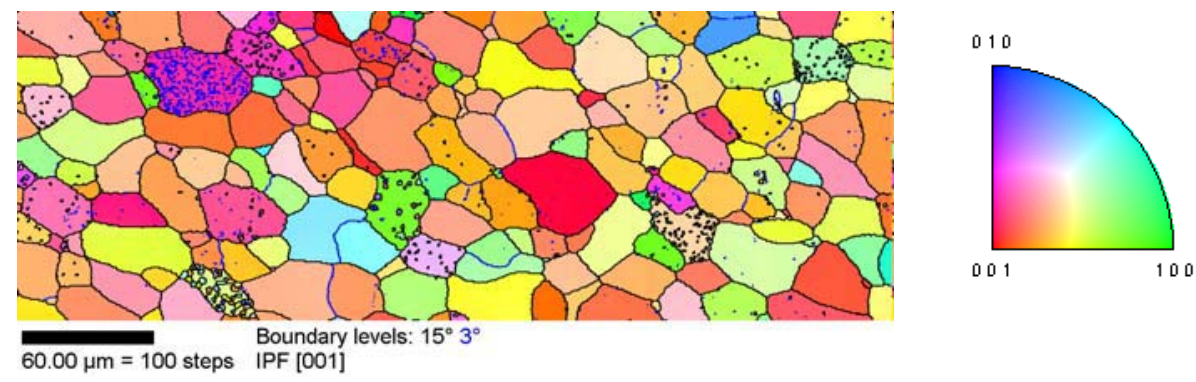

FIG. 2 IPF map of wrought $\alpha$-uranium showing grain structure and tendency toward (001) texture.
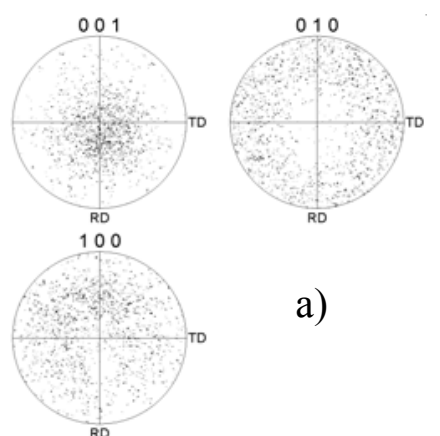

a)

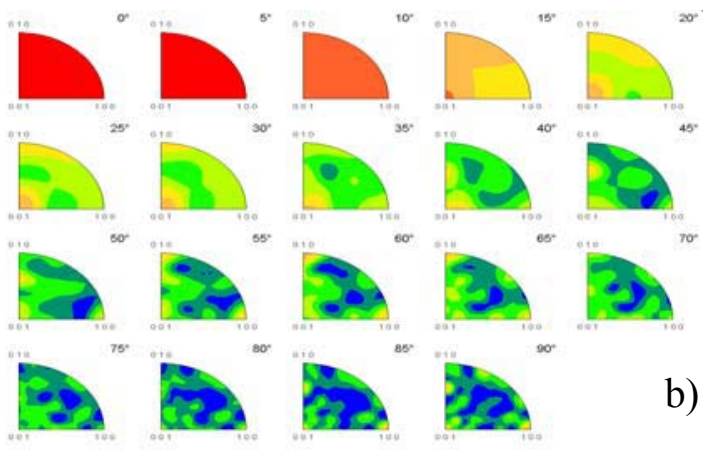

FIG. 3 a) Discrete pole figures of wrought $\alpha$-uranium showing (001) fiber texture. b) Texturereduced MDF showing peak at $60^{\circ} /[010]$. 